\title{
К проблеме выразимости операций характеристических матриц паранепротиворечивых и параполных логик
}

Н. А. ЗНАМЕНСКАЯ

\begin{abstract}
The following expressibilities are constructed: (1) implication of every logical matrix $\mathcal{M}_{P C o n t}, \mathcal{M}_{P C o n t(1)}$ and $\mathcal{M}_{P C o m p(1)}$ in terms of the operations of the logical matrix $\mathcal{M}_{L P F}$, (2) implication of every logical matrix $\mathcal{M}_{P C o n t}, \mathcal{M}_{L P F}$ and $\mathcal{M}_{P C o m p(1)}$ in terms of the operations of the logical matrix $\mathcal{M}_{P \text { Cont (1) }}$, (3) implication of every logical matrix $\mathcal{M}_{\text {PCont }}, \mathcal{M}_{L P F}$ and $\mathcal{M}_{P \operatorname{Cont}(1)}$ in terms of the operations of the logical matrix $\mathcal{M}_{P C o m p(1)}$. Also it's proven that the implications of the logical matrices $\mathcal{M}_{L P F}, \mathcal{M}_{P C o n t(1)}$ and $\mathcal{M}_{P C o m p(1)}$ are not expressible in terms of the operations of the logical matrix $\mathcal{M}_{\text {PCont }}$.
\end{abstract}

Keywords: matrix, operation, implication, valuation, expressibilitiy, paraconsistent logic, paracomplete logic

В [1] построена трехзначная характеристическая матрица для паранепротиворечивой логики PCont (обозначаем эту матрицу через $\left.\mathcal{M}_{\text {PCont }}\right)$, в [2] - трехзначная характеристическая матрица для параполной логики $\mathrm{LPF}^{1}$ (обозначаем эту матрицу через $\left.\mathcal{M}_{L P F}\right)$, в [3] - трехзначные характеристические матрицы для паранепротиворечивой логики PCont(1) (обозначаем эту матрицу через $\left.\mathcal{M}_{P \operatorname{Cont}(1)}\right)$ и параполной логики $\operatorname{PComp}(1)$ (обозначаем эту матрицу через $\left.\mathcal{M}_{P C o m p(1)}\right)$. В предлагаемой работе конструируются тождества, выражающие: 1) импликацию каждой матрицы $\mathcal{M}_{\text {PCont }}, \mathcal{M}_{\text {PCont(1) }}, \mathcal{M}_{\text {PComp(1) }}$ через операции матрицы $\left.\mathcal{M}_{L P F}, 2\right)$ импликацию каждой матрицы $\mathcal{M}_{P C o n t}, \mathcal{M}_{L P F}$, $\mathcal{M}_{P \operatorname{Comp}(1)}$ через операции матрицы $\left.\mathcal{M}_{P \operatorname{Cont}(1)}, 3\right)$ импликацию каждой матрицы $\mathcal{M}_{P C o n t}, \mathcal{M}_{L P F}, \mathcal{M}_{P \operatorname{Cont}(1)}$ через операции матрицы $\mathcal{M}_{P C o m p(1)}$. Кроме того, доказывается, что импликации

${ }^{1} \mathrm{~B}[4]$ и [5] логика LPF обозначена посредством PComp. 
логических матриц $\mathcal{M}_{L P F}, \mathcal{M}_{P C o n t(1)}$ и $\mathcal{M}_{P C o m p(1)}$ не являются выразимыми через операции матрицы $\mathcal{M}_{P C o n t}$.

Следуя [1], [2] и [3], воспроизведем (с точностью до обозначения элементов носителей матриц и матричных операций) определения этих логических матриц.

Характеристическая матрица из [1] для PCont есть логическая матрица $\left\langle\left\{1, \frac{1}{2}, 0\right\},\left\{1, \frac{1}{2}\right\},\left\{\neg, \&, \vee, \supset_{P C o n t}\right\}\right\rangle$, где

\begin{tabular}{|c|c|}
\hline & $\neg$ \\
\hline 1 & 0 \\
$\frac{1}{2}$ & $\frac{1}{2}$ \\
0 & 1 \\
\hline
\end{tabular}

\begin{tabular}{|c|ccc|}
\hline$\&$ & 1 & $\frac{1}{2}$ & 0 \\
\hline 1 & 1 & $\frac{1}{2}$ & 0 \\
$\frac{1}{2}$ & $\frac{1}{2}$ & $\frac{1}{2}$ & 0 \\
0 & 0 & 0 & 0 \\
\hline
\end{tabular}

\begin{tabular}{|c|ccc|}
\hline$\vee$ & 1 & $\frac{1}{2}$ & 0 \\
\hline 1 & 1 & 1 & 1 \\
$\frac{1}{2}$ & 1 & $\frac{1}{2}$ & $\frac{1}{2}$ \\
0 & 1 & $\frac{1}{2}$ & 0 \\
\hline
\end{tabular}

\begin{tabular}{|c|ccc|}
\hline$\supset_{\text {PCont }}$ & 1 & $\frac{1}{2}$ & 0 \\
\hline 1 & 1 & $\frac{1}{2}$ & 0 \\
$\frac{1}{2}$ & 1 & $\frac{1}{2}$ & 0 \\
0 & 1 & 1 & 1 \\
\hline
\end{tabular}

Характеристическая матрица из [2] для LPF есть логическая матрица $\left\langle\left\{1, \frac{1}{2}, 0\right\},\{1\},\left\{\neg, \boldsymbol{\&}, \boldsymbol{V}, \supset_{\boldsymbol{L P} \boldsymbol{F}}\right\}\right\rangle$. Характеристическая матрица из [3] для PCont(1) есть логическая матрица $\left\langle\left\{1, \frac{1}{2}, 0\right\}\right.$, $\left.\left\{1, \frac{1}{2}\right\},\left\{\neg, \mathbb{\&}, \vee, \supset_{\boldsymbol{P C o n t}(\mathbf{1})}\right\}\right\rangle$. Характеристическая матрица из [3] для $\mathrm{PComp}(1)$ есть логическая матрица $\left\langle\left\{1, \frac{1}{2}, 0\right\},\{1\},\{\neg, \&, \vee\right.$, $\left.\left.\supset_{P C o m p(1)}\right\}\right\rangle$. Операции $\supset_{L P F}, \supset_{P C o n t}(1), \supset_{P C o m p}(1)$ задаются следующими таблицами:

\begin{tabular}{|c|ccc|}
\hline$\supset_{\boldsymbol{L P F}}$ & 1 & $\frac{1}{2}$ & 0 \\
\hline 1 & 1 & $\frac{1}{2}$ & 0 \\
$\frac{1}{2}$ & 1 & 1 & 1 \\
0 & 1 & 1 & 1 \\
\hline
\end{tabular}

\begin{tabular}{|c|ccc|}
\hline$\supset_{\text {PCont (1) }}$ & 1 & $\frac{1}{2}$ & 0 \\
\hline 1 & 1 & 1 & 0 \\
$\frac{1}{2}$ & 1 & 1 & 0 \\
0 & 1 & 1 & 1 \\
\hline
\end{tabular}

\begin{tabular}{|c|ccc|}
\hline$\supset_{\text {PComp (1) }}$ & 1 & $\frac{1}{2}$ & 0 \\
\hline 1 & 1 & 0 & 0 \\
$\frac{1}{2}$ & 1 & 1 & 1 \\
0 & 1 & 1 & 1 \\
\hline
\end{tabular}

Существуют различные определения выразимости операций через операции. Здесь мы придерживаемся определений 1-3, предлагаемых В. М. Поповым. Условимся использовать символы $p_{1}, p_{2}, p_{3}, \ldots$ в качестве переменных, а символы $($,$) и , в ка-$ честве технических символов. Определения 1 и 2 , а также формулируемые далее условия предполагают, что $F_{1}, \ldots, F_{k}(k-$ целое положительное число) есть операции на непустом множестве $M$, а $s_{1}, \ldots, s_{k}$ есть попарно различные символы, каждый из которых отличен от любого из символов $(),,, p_{1}, p_{2}, p_{3}, \ldots$

ОПРЕДЕЛЕНИЕ $1 . F_{1} \ldots F_{k} s_{1} \ldots s_{k}$-формулы:

(1) для всякого целого положительного числа $n p_{n}$ есть $F_{1} \ldots$ $F_{k} s_{1} \ldots s_{k}$-формула, 
(2) если $i \in\{1, \ldots, k\}$ и ранг операции $F_{i}$ есть 0 , то $s_{i}$ есть $F_{1} \ldots F_{k} s_{1} \ldots s_{k}$-формула,

(3) если $i \in\{1, \ldots, k\}$ и ранг операции $F_{i}$ отличен от 0 и равен $l$, а $A_{1}, \ldots, A_{l}$ есть $F_{1} \ldots F_{k} s_{1} \ldots s_{k}$-формулы, то $s_{i}\left(A_{1}, \ldots\right.$, $A_{l}$ ) есть $F_{1} \ldots F_{k} s_{1} \ldots s_{k}$-формула,

(4) ничто другое не есть $F_{1} \ldots F_{k} s_{1} \ldots s_{k}$-формула.

ОПРЕДЕЛЕНИЕ $2 . F_{1} \ldots F_{k} s_{1} \ldots s_{k}$-оценки:

$F_{1} \ldots F_{k} s_{1} \ldots s_{k}$-оценкой называем отображение множества всех переменных во множество $M$.

Можно доказать, что существует единственное отображение, обозначим его через $\|\left.\right|^{F_{1} \ldots F_{k} s_{1} \ldots s_{k}}$, удовлетворяющее условиям:

(1) ||$^{F_{1} \ldots F_{k} s_{1} \ldots s_{k}}$ есть отображение в $M$ декартова произведения множества всех $F_{1} \ldots F_{k} s_{1} \ldots s_{k}$-формул на множество всех $F_{1} \ldots F_{k} s_{1} \ldots s_{k}$-оценок,

(2) если $n$ есть целое положительное число и $v$ есть $F_{1} \ldots$ $F_{k} s_{1} \ldots s_{k}$-оценка, то $\left|p_{n}, v\right|^{F_{1} \ldots F_{k} s_{1} \ldots s_{k}}=v\left(p_{n}\right)$,

(3) если $i \in\{1, \ldots, k\}$, ранг операции $F_{i}$ есть 0 и $v$ есть $F_{1} \ldots$ $F_{k} s_{1} \ldots s_{k}$-оценка, то $\left|s_{i}, v\right|^{F_{1} \ldots F_{k} s_{1} \ldots s_{k}}=F_{i}$,

(4) если $i \in\{1, \ldots, k\}$, ранг операции $F_{i}$ отличен от 0 и равен $l, A_{1}, \ldots, A_{l}$ есть $F_{1} \ldots F_{k} s_{1} \ldots s_{k}$-формулы и $v$ есть $F_{1} \ldots F_{k} s_{1} \ldots s_{k}$-оценка, то $\left|s_{i}\left(A_{1}, \ldots, A_{l}\right), v\right|^{F_{1} \ldots F_{k} s_{1} \ldots s_{k}}=$ $F_{i}\left(\left|A_{1}, v\right|^{F_{1} \ldots F_{k} s_{1} \ldots s_{k}}, \ldots,\left|A_{l}, v\right|^{F_{1} \ldots F_{k} s_{1} \ldots s_{k}}\right)$.

ОПРЕДЕЛЕНИЕ 3. Операция $g$ выразима через операции $f_{1}, \ldots, f_{k}$ ( $k$ - целое положительное число), если операции $g$, $f_{1}, \ldots, f_{k}$ являются операциями на одном и том же непустом множестве, существуют попарно различные символы $s_{1}, \ldots, s_{k}$, каждый из которых отличен от любого из символов $(),,,, p_{1}, p_{2}$, $p_{3}, \ldots$ и существует такая $f_{1} \ldots f_{k} s_{1} \ldots s_{k}$-формула $\Phi$, что выполняются следующие условия:

(1) если ранг операции $g$ равен 0 , то для всякой $f_{1} \ldots f_{k} s_{1} \ldots s_{k^{-}}$ оценки $v g=|\Phi, v|^{f_{1} \ldots f_{k} s_{1} \ldots s_{k}}$, 
(2) если ранг операции $g$ есть целое положительное число $n$, то для всякой $f_{1} \ldots f_{k} s_{1} \ldots s_{k}$-оценки $v$ верно, что $g\left(v\left(p_{1}\right), \ldots\right.$, $\left.v\left(p_{n}\right)\right)=\left.|\Phi, v|\right|^{f_{1} \ldots f_{k} s_{1} \ldots s_{k}}$.

Очевидны следующие утверждения (1)-(4).

(1) Символы $\neg, \&, \vee, \supset$ попарно различны и каждый из них отличен от любого из символов $(),,,, p_{1}, p_{2}, p_{3}, \ldots$

(2) Операции $\neg, \&, \vee, \supset_{P C o n t}, \supset_{L P F}, \supset_{P C o n t(1)}, \supset_{P C o m p(1)}$ являются операциями на одном и том же множестве (на множестве $\left.\left\{1, \frac{1}{2}, 0\right\}\right)$.

(3) $\neg p_{1}, p_{1} \& p_{2}, p_{1} \vee p_{2}$ и $p_{1} \supset p_{2}$ есть $\neg \& \vee \supset \boldsymbol{L P F} \neg \& \vee \supset$ формулы, $\neg \& \vee \supset_{P \operatorname{Cont}(1)} \neg \& \vee \supset$-формулы и $\neg \& \vee$ $\supset_{P C o m p(1)} \neg \& \bigvee \supset_{\text {-формулы }}^{2}$ (внешние скобки опускаем, как это принято).

(4) Если $f_{1}, \ldots, f_{k}$ ( $k$ - целое положительное число) суть операции на некотором непустом множестве, то для всякого $i$ ( $i$ есть целое положительное число, меньшее или равное $k$ ) операция $f_{i}$ выразима через операции $f_{1}, \ldots, f_{k}$.

TEOPEMA 1 (о выразимости, операций матриц $\mathcal{M}_{P C o n t}$, $\mathcal{M}_{P \operatorname{Cont}(1)}$ и $\mathcal{M}_{P \operatorname{Comp}(1)}$ через операции матрицы $\left.\mathcal{M}_{L P F}\right)$.

Всякая операчия $\neg, \&, \vee, \supset_{P C o n t}, \supset_{P C o n t}(1), \supset_{P C o m p(1)}$ выразима через операчии $\neg, \&, \vee, \supset_{\boldsymbol{L P F}}$.

Доказательство. В свете утверждений (1)-(4) и определения 3 понятно, что для доказательства Теоремы 1 достаточно доказать утверждение (5).

(5) Для всякой $\neg \& \vee \supset_{\boldsymbol{L P F}} \neg \& \vee \supset$-оценки $v: v\left(p_{1}\right) \supset_{\boldsymbol{P C o n t}}$ $v\left(p_{2}\right)=\left|\left(\neg p_{1} \supset p_{2}\right) \supset p_{2}, v\right| \neg \& \vee \supset_{L P F} \neg \& \vee \supset, v\left(p_{1}\right) \supset_{P C \operatorname{Cont}(1)}$ $v\left(p_{2}\right)=\left|\neg\left(\left(\neg p_{2} \supset \neg p_{1}\right) \supset \neg\left(p_{1} \supset p_{1}\right)\right), v\right| \neg \& \vee \supset_{L P F} \neg \& \vee \supset$, $v\left(p_{1}\right) \supset_{\boldsymbol{P C o m p}(\mathbf{1})} v\left(p_{2}\right)=\mid \neg\left(\left(p_{1} \supset p_{2}\right) \supset \neg\left(p_{1} \supset p_{1}\right)\right)$, $v \mid \neg \& \vee \supset_{L P F} \neg \& \vee \supset$.

\footnotetext{
${ }^{2}$ Автор надеется, что естественное для данного контекста использование инфиксной, а не префиксной, записи не создаст трудности для читателя.
} 
Докажем утверждение (5).

(5.1) $v_{0}$ есть $\neg \& \vee \supset_{\boldsymbol{L P F}} \neg \& \vee \supset$-оценка (допущение).

Используя утверждение (5.1) и определение отображения

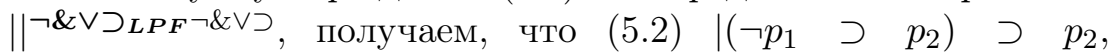
$\left.v_{0}\right|^{\neg \& \vee \supset_{L P F} \neg \& \vee \supset}=\left(\begin{array}{lllll}\neg v_{0}\left(p_{1}\right) & \supset_{\boldsymbol{L P F}} & v_{0}\left(p_{2}\right)\end{array}\right) \supset_{\boldsymbol{L P F}} \quad v_{0}\left(p_{2}\right)$, $\left|\neg\left(\left(\neg p_{2} \quad \supset \quad \neg p_{1}\right) \quad \supset \quad \neg\left(p_{1} \quad \supset \quad p_{1}\right)\right), v_{0}\right| \neg \& \vee \supset_{L P F} \neg \& \vee \supset=$ $\neg\left(\left(\neg v_{0}\left(p_{2}\right) \quad \supset_{\boldsymbol{L P F}} \quad \neg v_{0}\left(p_{1}\right)\right) \quad \supset_{\boldsymbol{L P F}} \quad \neg\left(v_{0}\left(p_{1}\right) \quad \supset_{\boldsymbol{L P F}} \quad v_{0}\left(p_{1}\right)\right)\right)$, $\left|\neg\left(\left(p_{1} \supset p_{2}\right) \supset \neg\left(p_{1} \supset p_{1}\right)\right), v_{0}\right| \neg \& \vee \supset_{L P F} \neg \& \vee \supset=\neg\left(\left(v_{0}\left(p_{1}\right) \supset_{L P F}\right.\right.$ $\left.\left.v_{0}\left(p_{2}\right)\right) \supset_{\boldsymbol{L P F}} \neg\left(v_{0}\left(p_{1}\right) \supset_{\boldsymbol{L P F}} v_{0}\left(p_{1}\right)\right)\right)$.

Опираясь на табличные определения операций $\supset_{\boldsymbol{P C o n t}}$, $\supset_{P C o n t(1)}, \supset_{P C o m p(1)}, \neg$ и $\supset_{\boldsymbol{L P F}}$, получаем, что

$(5.3) v_{0}\left(p_{1}\right) \supset_{\boldsymbol{P C o n t}} v_{0}\left(p_{2}\right)=\left(\neg v_{0}\left(p_{1}\right) \supset_{\boldsymbol{L P F}} v_{0}\left(p_{2}\right)\right) \supset_{\boldsymbol{L P F}}$ $v_{0}\left(p_{2}\right), v_{0}\left(p_{1}\right) \supset_{\boldsymbol{P C o n t}(1)} v_{0}\left(p_{2}\right)=\neg\left(\left(\neg v_{0}\left(p_{2}\right) \supset_{\boldsymbol{L P F}} \neg v_{0}\left(p_{1}\right)\right) \supset_{\boldsymbol{L P F}}\right.$ $\left.\neg\left(v_{0}\left(p_{1}\right) \supset_{\boldsymbol{L P F}} v_{0}\left(p_{1}\right)\right)\right), v_{0}\left(p_{1}\right) \supset_{\boldsymbol{P C o m p}(1)} v_{0}\left(p_{2}\right)=\neg\left(\left(v_{0}\left(p_{1}\right) \supset_{\boldsymbol{L P} \boldsymbol{F}}\right.\right.$ $\left.\left.v_{0}\left(p_{2}\right)\right) \supset_{\boldsymbol{L} \boldsymbol{P} \boldsymbol{F}} \neg\left(v_{0}\left(p_{1}\right) \supset_{\boldsymbol{L P} \boldsymbol{F}} v_{0}\left(p_{1}\right)\right)\right)$.

(5.4) $v_{0}\left(p_{1}\right) \supset_{\text {PCont }} v_{0}\left(p_{2}\right)=\left|\left(\neg p_{1} \supset p_{2}\right) \supset p_{2}, v_{0}\right|^{\neg \& \vee \supset_{L P F} \neg \& \vee \supset}$, $v_{0}\left(p_{1}\right) \supset_{\text {PCont }}(\mathbf{1}) \quad v_{0}\left(p_{2}\right)=\mid \neg\left(\left(\neg p_{2} \supset \neg p_{1}\right) \supset \neg\left(p_{1} \supset p_{1}\right)\right)$, $v_{0}\left|\neg \& \vee \supset_{L P F} \sim \& \vee \supset, v_{0}\left(p_{1}\right) \supset_{\boldsymbol{P C o m p}(1)} v_{0}\left(p_{2}\right)=\right| \neg\left(\left(p_{1} \supset p_{2}\right) \supset\right.$ $\left.\neg\left(p_{1} \supset p_{1}\right)\right),\left.v_{0}\right|^{\neg \& \vee \supset \boldsymbol{L P F} \neg \& \vee \supset}$ (из (5.2) и (5.3)).

Снимая допущение (5.1) и обобщая, получаем, что верно утверждение (5).

Q.E.D.

TEOPEMA 2 (о выразимости операций матриц $\mathcal{M}_{P C o n t}, \mathcal{M}_{L P F}$ и $\mathcal{M}_{P \operatorname{Comp}(1)}$ через операции матрицы $\left.\mathcal{M}_{P \operatorname{Cont}(1)}\right)$.

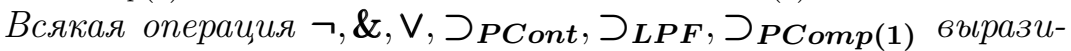
ма через операчии $\neg, \&, \vee, \supset_{P C \operatorname{Cont}(1)}$.

Доказательство. В свете утверждений (1)-(4) и определения 3 понятно, что для доказательства Теоремы 2 достаточно доказать утверждение (6).

(6) Для всякой $\neg \& \vee \supset_{\boldsymbol{P C o n t}(1)} \neg \& \vee \supset$-оценки $v: v\left(p_{1}\right) \supset_{\boldsymbol{P C o n t}}$ $v\left(p_{2}\right)=\left|\left(p_{1} \supset \neg\left(\left(p_{2} \supset p_{1}\right) \supset p_{1}\right)\right) \vee p_{2}, v\right| \neg \& \vee \supset P_{P \operatorname{Cont}(1)} \neg \& \vee \supset$, $v\left(p_{1}\right) \supset_{\boldsymbol{L P F}} v\left(p_{2}\right)=\left|\left(p_{1} \supset \neg p_{1}\right) \vee p_{2}, v\right| \neg \& \vee \supset_{P \operatorname{Cont}(1)} \neg \& \vee \supset$, $v\left(p_{1}\right) \supset_{\boldsymbol{P C o m p}(1)} v\left(p_{2}\right)=\left|\neg p_{2} \supset \neg p_{1}, v\right| \neg \& \vee \supset_{P \operatorname{Cont}(1)} \neg \& \vee \supset$.

Докажем утверждение (6).

(6.1) $v_{0}$ есть $\neg \& \vee \supset_{P \operatorname{Cont}(1)} \neg \& \vee \supset$-оценка (допущение). 
Используя утверждение (6.1) и определение отображения $\| \neg \& \vee \supset_{\text {PCont (1) }} \neg \& \vee \supset$, получаем, что (6.2) $\mid\left(p_{1} \supset \neg\left(\left(p_{2} \supset p_{1}\right) \supset\right.\right.$ $\left.\left.p_{1}\right)\right) \vee p_{2},\left.v_{0}\right|^{\neg \& \vee \supset_{P C o n t(1)} \neg \& \vee \supset}=\left(v_{0}\left(p_{1}\right) \supset_{P C \operatorname{Cont}(1)} \neg\left(\left(v_{0}\left(p_{2}\right)\right.\right.\right.$ $\left.\left.\left.\supset_{\boldsymbol{P C o n t}(1)} v_{0}\left(p_{1}\right)\right) \supset_{\boldsymbol{P C O n t}(1)} v_{0}\left(p_{1}\right)\right)\right) \vee v_{0}\left(p_{2}\right), \mid\left(p_{1} \supset \neg p_{1}\right) \vee$ $p_{2},\left.v_{0}\right|^{\neg \& \vee \supset_{P C o n t}(1) \neg \& \vee \supset}=\left(v_{0}\left(p_{1}\right) \supset_{P C \operatorname{Cont}(1)} \neg v_{0}\left(p_{1}\right)\right) \vee v_{0}\left(p_{2}\right)$, $\left|\neg p_{2} \supset \neg p_{1}, v_{0}\right|^{\neg \& \vee \supset P_{\text {Cont }(1)} \neg \& \vee \supset}=\neg v_{0}\left(p_{2}\right) \supset_{P \text { Cont }(1)} \neg v_{0}\left(p_{1}\right)$.

Опираясь на табличные определения операций $\supset_{\boldsymbol{P C} \text { cont }}, \supset_{\boldsymbol{L P F}}$, $\supset_{P \text { Comp }(1)}, \neg, \vee$ и $\supset_{P \text { Cont }(1)}$, получаем, что

$(6.3) v_{0}\left(p_{1}\right) \supset_{\text {PCont }} v_{0}\left(p_{2}\right)=\left(v_{0}\left(p_{1}\right) \supset_{\text {PCont }(1)} \neg\left(\left(v_{0}\left(p_{2}\right)\right.\right.\right.$ $\left.\left.\left.\supset_{P C o n t}(1) \quad v_{0}\left(p_{1}\right)\right) \supset_{\boldsymbol{P C o n t}}(\mathbf{1}) \quad v_{0}\left(p_{1}\right)\right)\right) \vee v_{0}\left(p_{2}\right), v_{0}\left(p_{1}\right) \supset_{\boldsymbol{L P F}}$ $v_{0}\left(p_{2}\right)=\left(v_{0}\left(p_{1}\right) \supset_{P \operatorname{Cont}(1)} \neg v_{0}\left(p_{1}\right)\right) \vee v_{0}\left(p_{2}\right), v_{0}\left(p_{1}\right) \supset_{\boldsymbol{P C o m p}(1)}$ $v_{0}\left(p_{2}\right)=\neg v_{0}\left(p_{2}\right) \supset_{P \text { Cont }}(\mathbf{1}) \neg v_{0}\left(p_{1}\right)$.

(6.4) $v_{0}\left(p_{1}\right) \supset_{\text {PCont }} v_{0}\left(p_{2}\right)=\mid\left(p_{1} \supset \neg\left(\left(p_{2} \supset p_{1}\right) \supset p_{1}\right)\right) \vee$ $p_{2}, v_{0}\left|\neg \& \vee \supset_{P \operatorname{Cont}(1)} \backsim \& \vee \supset, v_{0}\left(p_{1}\right) \supset_{\boldsymbol{L P F}} \quad v_{0}\left(p_{2}\right)=\right|\left(p_{1} \supset \neg p_{1}\right) \vee$ $p_{2}, v_{0}\left|\neg \& \vee \supset_{P C o n t(1)} \neg \& \vee \supset, v_{0}\left(p_{1}\right) \supset_{P C o m p(1)} v_{0}\left(p_{2}\right)=\right| \neg p_{2} \supset \neg p_{1}$, $v_{0} \mid \neg \& \vee \supset_{\text {PCont (1) }} \neg \& \vee \supset$ (из (6.2) и (6.3)).

Снимая допущение (6.1) и обобщая, получаем, что верно утверждение (6).

Q.E.D.

TEOPEMA 3 (о выразимости операций матриц $\mathcal{M}_{\text {PCont }}, \mathcal{M}_{L P F}$ и $\mathcal{M}_{P \operatorname{Cont}(1)}$ через операции матрицы $\left.\mathcal{M}_{P \operatorname{Comp}(1)}\right)$.

Всякая операчия $\neg, \&, \vee, \supset_{P C o n t}, \supset_{L P F}, \supset_{P C o n t}(1)$ выразима через операчии $\neg, \&, \vee, \supset_{\boldsymbol{P C} \boldsymbol{C} \boldsymbol{p}(\mathbf{1})}$.

Доказательство. В свете утверждений (1)-(4) и определения 3 понятно, что для доказательства Теоремы 3 достаточно доказать утверждение (7).

(7) Для всякой $\neg \& \vee \supset_{P C o m p(1)} \neg \& \vee \supset$-оценки $v: v\left(p_{1}\right) \supset_{P C o n t}$ $v\left(p_{2}\right)=\left|\left(\left(p_{2} \supset p_{1}\right) \supset \neg p_{1}\right) \vee p_{2}, v\right| \neg \& \vee \supset_{P C o m p(1)} \neg \& \vee \supset$, $v\left(p_{1}\right) \supset_{\boldsymbol{L P F}} v\left(p_{2}\right)=\left|\left(p_{1} \supset p_{2}\right) \vee p_{2}, v\right| \neg \& \vee \supset_{P C o m p(1)} \neg \& \vee \supset$, $v\left(p_{1}\right) \supset_{\boldsymbol{P C O n t}(1)} v\left(p_{2}\right)=\left|\neg p_{2} \supset \neg p_{1}, v\right| \neg \& \vee \supset_{P C o m p(1)} \neg \& \vee \supset$.

Докажем утверждение (7).

(7.1) $v_{0}$ есть $\neg \& \vee \supset \boldsymbol{P C o m p ( 1 )} \neg \& \vee \supset$-оценка (допущение).

Используя утверждение (7.1) и определение отображения $\| \neg \& \vee \supset_{P \text { Cотр }(1) \neg \& \vee \supset}$, получаем, что $(7.2) \mid\left(\left(p_{2} \supset p_{1}\right) \supset \neg p_{1}\right) \vee$ $p_{2},\left.v_{0}\right|^{\neg \& \vee \supset_{P \operatorname{Comp}(1)} \neg \& \vee \supset}=\left(\left(v_{0}\left(p_{2}\right) \supset_{\boldsymbol{P C O m p}(1)} v_{0}\left(p_{1}\right)\right) \supset_{\boldsymbol{P C o m p}(1)}\right.$ 
$\left.\neg v_{0}\left(p_{1}\right)\right) \vee v_{0}\left(p_{2}\right),\left|\left(p_{1} \supset p_{2}\right) \vee p_{2}, v_{0}\right|^{\neg \& \vee \supset \supset_{P C o m p(1)} \neg \& \vee \supset}=\left(v_{0}\left(p_{1}\right)\right.$ $\left.\supset_{P C o m p(1)} v_{0}\left(p_{2}\right)\right) \vee v_{0}\left(p_{2}\right),\left|\neg p_{2} \supset \neg p_{1}, v_{0}\right| \neg \& \vee \supset_{P C o m p(1)} \neg \& \vee \supset=$ $\neg v_{0}\left(p_{2}\right) \supset_{\boldsymbol{P C} \operatorname{comp}(1)} \neg v_{0}\left(p_{1}\right)$.

Опираясь на табличные определения операций $\supset_{\boldsymbol{P C o n t}}, \supset_{\boldsymbol{L P F}}$, $\supset_{\boldsymbol{P C o n t}(1)}, \neg, \vee$ и $\supset_{\boldsymbol{P C o m p}(1)}$, получаем, что

$(7.3) \quad v_{0}\left(p_{1}\right)$ つPCont $v_{0}\left(p_{2}\right)=\left(\left(v_{0}\left(p_{2}\right) \supset_{\boldsymbol{P C o m p}(1)} v_{0}\left(p_{1}\right)\right)\right.$ $\left.\supset_{\boldsymbol{P C o m p}(1)} \neg v_{0}\left(p_{1}\right)\right) \vee v_{0}\left(p_{2}\right), v_{0}\left(p_{1}\right) \supset_{\boldsymbol{L P F}} \quad v_{0}\left(p_{2}\right)=\left(v_{0}\left(p_{1}\right)\right.$ $\left.\supset_{P C o m p(1)} v_{0}\left(p_{2}\right)\right) \vee v_{0}\left(p_{2}\right), v_{0}\left(p_{1}\right) \supset_{P C \operatorname{Cont}(1)} v_{0}\left(p_{2}\right)=\neg v_{0}\left(p_{2}\right)$ $\supset_{P C o m p(1)} \neg v_{0}\left(p_{1}\right)$.

$(7.4) v_{0}\left(p_{1}\right) \supset$ PCont $v_{0}\left(p_{2}\right)=\mid\left(\left(p_{2} \supset p_{1}\right) \supset \neg p_{1}\right) \vee p_{2}$, $v_{0}\left|\neg \& \vee \supset_{P C o m p(1) \neg \& \vee \supset}, v_{0}\left(p_{1}\right) \supset_{\boldsymbol{L P F}} \quad v_{0}\left(p_{2}\right)=\right|\left(p_{1} \supset p_{2}\right) \vee p_{2}$, $\left.v_{0}\right|^{\neg \& \vee \supset_{P \operatorname{Comp}(1)} \neg \& \vee \supset}, v_{0}\left(p_{1}\right) \supset_{P \operatorname{Cont}(1)} v_{0}\left(p_{2}\right)=\mid \neg p_{2} \supset \neg p_{1}$, $v_{0} \mid \neg \& \vee \supset_{P C o m p(1)} \neg \& \vee \supset$ (из (7.2) и (7.3)).

Снимая допущение (7.1) и обобщая, получаем, что верно утверждение (7).

Q.E.D.

ЛЕММА. Пусть $s_{1}, s_{2}, s_{3}$ и $s_{4}$ есть попарно различные символы, каждый из которых отличен от любого из символов $(),,,, p_{1}, p_{2}$, $p_{3}, \ldots$, а $v$ есть такая $\neg \& \vee \supset_{\boldsymbol{P C o n t}} s_{1} s_{2} s_{3} s_{4}$-оценка, что $v\left(p_{i}\right)=$ $\frac{1}{2}$ для всякого целого положительного числа $i$.

Для всякой $\neg \& \vee \supset_{\text {PCont }} s_{1} s_{2} s_{3} s_{4}$-формулы $A$ верно, что $|A, v| \neg \& \vee \supset_{P C o n t} s_{1} s_{2} s_{3} s_{4}=\frac{1}{2}$.

Лемма доказывается индукцией по построению $\neg \& \vee \supset$ PCont $s_{1} s_{2} s_{3} s_{4}$-формулы.

TEOPEMA 4 (о невыразимости $\supset_{\boldsymbol{L P F}}, \supset_{\boldsymbol{P C o n t}(1)}$ и $\supset_{\boldsymbol{P C o m p}(1)}$ через операции матрицы $\left.\mathcal{M}_{P C o n t}\right)$.

Onерачии $\supset_{\boldsymbol{L P F}}, \supset_{\boldsymbol{P C o n t}(1)} u \supset_{\boldsymbol{P C o m p}(1)}$ не являются выразимыми через операции $\neg, \&, \vee, \supset$ PCont.

Доказательство. Доказажем, что $\supset_{\boldsymbol{L P F}}$ не является выразимой через операции $\neg, \&, \vee, \supset$ PCont.

Доказательство проводим от противного.

(1) $\supset_{\boldsymbol{L P F}}$ выразима через $\neg, \boldsymbol{\&}, \vee, \supset_{\boldsymbol{P C} \text { ont }}$ (допущение).

(2) Существуют попарно различные символы $s_{1}, s_{2}, s_{3}, s_{4}$, каждый из которых отличен от любого из символов $(),,,, p_{1}, p_{2}, p_{3}, \ldots$ и существует такая $\neg \& \bigvee \supset_{\text {PCont }} s_{1} s_{2} s_{3} s_{4}$-формула $\Phi$, что для 
всякой $\neg \& \vee \supset$ PCont $s_{1} s_{2} s_{3} s_{4}$-оценки $v$ верно, что $v\left(p_{1}\right) \supset_{\boldsymbol{L P F}}$ $v\left(p_{2}\right)=|\Phi, v| \neg \& \vee \supset \boldsymbol{P C o n t}_{1} s_{2} s_{2} s_{3} s_{4}$ (из (1), по определению 3).

Пусть (3) $s_{1}^{\prime}, s_{2}^{\prime}, s_{3}^{\prime}, s_{4}^{\prime}$ есть попарно различные символы, каждый из которых отличен от любого из символов $(),,,, p_{1}, p_{2}$, $p_{3}, \ldots$, а $\Phi^{\prime}$ есть такая $\neg \& \vee \supset_{\boldsymbol{P C o n t}} s_{1}^{\prime} s_{2}^{\prime} s_{3}^{\prime} s_{4}^{\prime}$-формула, что для всякой $\neg \& \vee \supset_{\boldsymbol{P C} \text { Cont }} s_{1}^{\prime} s_{2}^{\prime} s_{3}^{\prime} s_{4}^{\prime}$-оценки $v$ верно, что $v\left(p_{1}\right) \supset_{\boldsymbol{L P} \boldsymbol{F}}$ $v\left(p_{2}\right)=\left|\Phi^{\prime}, v\right| \neg \& \vee \supset P_{\text {Pont }} s_{1}^{\prime} s_{2}^{\prime} s_{3}^{\prime} s_{4}^{\prime}$.

Очевидно, что (4) существует такая $\neg \& \vee \supset_{\boldsymbol{P C o n t}} s_{1}^{\prime} s_{2}^{\prime} s_{3}^{\prime} s_{4^{-}}^{\prime}$ оценка $v$, что $v\left(p_{i}\right)=\frac{1}{2}$ для всякого целого положительного числа $i$.

Пусть (5) $v^{\prime}$ есть такая $\neg \& \vee \supset_{\boldsymbol{P C o n t}} s_{1}^{\prime} s_{2}^{\prime} s_{3}^{\prime} s_{4}^{\prime}$-оценка, что $v^{\prime}\left(p_{i}\right)=\frac{1}{2}$ для всякого целого положительного числа $i$.

(6) $\left|\Phi^{\prime}, v^{\prime}\right| \neg \& \vee \supset_{\text {PCont }} s_{1}^{\prime} s_{2}^{\prime} s_{3}^{\prime} s_{4}^{\prime}=\frac{1}{2}$ (из (3) и (5), по Лемме).

(7) $v^{\prime}\left(p_{1}\right) \supset_{\boldsymbol{L P F}} v^{\prime}\left(p_{2}\right)=\left|\Phi^{\prime}, v^{\prime}\right| \neg \& \vee \supset_{P C o n t} s_{1}^{\prime} s_{2}^{\prime} s_{3}^{\prime} s_{4}^{\prime}$ (из (3) и (5)).

Опираясь на утверждения (6) и (7), получаем, что

(8) $v^{\prime}\left(p_{1}\right) \supset_{\boldsymbol{L P F}} v^{\prime}\left(p_{2}\right) \neq 1$.

(9) $v^{\prime}\left(p_{1}\right) \supset_{\boldsymbol{L P F}} v^{\prime}\left(p_{2}\right)=1$ (из того, что $v^{\prime}\left(p_{1}\right)=v^{\prime}\left(p_{2}\right)=\frac{1}{2}$, по определению $\left.\supset_{\boldsymbol{L P F}}\right)$.

Утверждение (9) противоречит утверждению (8). Следовательно, неверно допущение (1). Но тогда $\supset_{\boldsymbol{L} \boldsymbol{P} \boldsymbol{F}}$ не является выразимой через $\neg, \&, \vee, \supset_{\text {PCont }}$.

Доказательство того, что $\supset_{\boldsymbol{P C o n t}(1)}$ не является выразимой через операции $\neg, \&, \vee, \supset$ PCont, и доказательство того, что $\supset_{\boldsymbol{P C o m p}(1)}$ не является выразимой через операции $\neg, \&, \vee$, $\supset_{\text {PCont }}$, аналогичны данному выше доказательству того, что $\supset_{\boldsymbol{L P F}}$ не является выразимой через операции $\neg, \boldsymbol{\&}, \vee, \supset$ PCont.

Q.E.D.

Автор выражает благодарность В. М. Попову за постановку проблемы и сделанные им исправления в черновом варианте статьи и А. С. Карпенко, прорецензировавшему статью и сообщившему, что уже в работе [6] приведены те же, что и в настоящей статье, тождества, выражающие импликацию $\supset_{\boldsymbol{P C o n t}(1)}$ через операции матрицы $\mathcal{M}_{P C o m p(1)}$ и импликацию $\supset_{\boldsymbol{P C o m p}(1)}$ через операции матрицы $\mathcal{M}_{P \operatorname{cont}(1)}$ (в целях сохранения целостности изложения мы не стали опускать здесь обоснование указанных тождеств). 


\section{Литература}

[1] Розоноэр Л. И. О выявлении противоречий в формальных теориях. 1 // Автоматика и телемеханика, № 6,1983 . С. 113-124.

[2] Avron A. Natural 3-valued Logics: Characterization and proof theory // Journal of Symbolic Logic. Vol. 56. 1991. P. 276-294

[3] Попов В. М. Между $\operatorname{Par}(1)$ и множеством всех формул // Объединенный научный журнал. № 7-8 (254-255). М., 2011. С. 35-39.

[4] Попов В. М. Между Раг и множеством всех формул // Материалы 6 конференции «Смирновские чтения по логике 2009». С. 93-95.

[5] Знаменская Н. А., Попов В. М. Паранормальная логика PContPComp как пересечение паранепротиворечивой логики PCont и параполной логики PComp // Материалы 6 конференции «Смирновские чтения по логике 2009». С.63-65.

[6] Томова Н. Е. Импликативные расширения регулярных логик Клини // Логические исследования. Вып. 16. М.-СПб., 2010. С.63-65. 